\title{
Invisible Thin-Film Patterns with Strong Infrared Emission as an Optical Security Feature
}

\author{
Gokhan Bakan, * Sencer Ayas, Murat Serhatlioglu, Caglar Elbuken, and Aykutlu Dana
}

Spectrally selective thermal emission is in high demand for

thermophotovoltaics, radiative cooling, and infrared sensing applications. Spectral control of the emissivity is historically achieved by choosing the material with suitable infrared properties. The recent advancements in nanofabrication techniques that lead to enhanced light-matter interactions enable optical properties like infrared emissivity that are not naturally available. In this study, thermal emitters based on nanometer-thick dielectrics on field-enhancement surfaces as optical security features are proposed.

Such a function is achieved by generating patterns by ultrathin dielectrics that are transparent in the visible and exhibit strong infrared absorption in the spectral range of thermal cameras. The invisible patterns are then revealed by thermal imaging. The field-enhancement surfaces enhance the emissivity of the patterns, in turn reduce the minimum temperature to detect the thermal emission down to $\approx 30^{\circ} \mathrm{C}$ from $>150^{\circ} \mathrm{C}$ to exploit ubiquitous heat sources like the human body. The study provides a framework for the use of thermal emitters as optical security features and demonstrates applications on rigid and flexible substrates.

\section{Introduction}

Hot bodies radiate electromagnetic waves mainly in the infrared and visible ranges of the electromagnetic spectrum. The radiation spectrum is broad and its intensity increases with increasing surface temperature while the peak intensity

Prof. G. Bakan, Dr. S. Ayas, M. Serhatlioglu, Prof. C. Elbuken, Dr. A. Dana UNAM Institute of Material Science and Nanotechnology

Bilkent University

Ankara 06800, Turkey

E-mail: gokhan.bakan@atilim.edu.tr

Prof. G. Bakan

Department of Electrical and Electronics Engineering

Atilim University

Ankara 06830, Turkey

Dr. S. Ayas

Canary Center at Stanford for Cancer Early Detection

Department of Radiology

Stanford School of Medicine

Palo Alto, CA 94304, USA

Dr. A. Dana

E. L. Ginzton Laboratory

Stanford University

Stanford, CA 94305, USA

The ORCID identification number(s) for the author(s) of this article can be found under https://doi.org/10.1002/adom.201800613.

DOI: 10.1002/adom.201800613 blueshifts as described by the Planck's law. The broad spectrum is useful for illumination purposes in the visible and as well as in the infrared. On the other hand, spectrally selective thermal emission is desired for applications like thermophotovoltaics, ${ }^{[1-3]}$ radiative cooling, ${ }^{[4,5]}$ sensing, ${ }^{[6-8]}$ and near-infrared communication $^{[9,10]}$ by modifying the emissivity of the surfaces. The emissivity of a surface is equal to its absorbance in thermal equilibrium according to the Kirchhoff's law of thermal emission, ranging from 0 for a perfect mirror to 1 for a perfect absorber or a blackbody. A simple approach to modify the emissivity is finding a dielectric with phonon bands at the desired absorption wavelength range. A recent example is embedding glass microspheres in an infrared transparent polymer matrix for radiative cooling. ${ }^{[11]}$ The dielectric dimensions can be as thin as micrometers when a back mirror, typically an optically thick metal layer is employed. This approach has been preferred for selective thermal emission since early 1980 s, e.g., using $\approx 1 \mu \mathrm{m}$ thick silicon monoxide on $\mathrm{Al}$ for radiative cooling. Owing to the simplicity of the structure, surfaces consisting of polar-dielectric-coated mirrors are still popular. ${ }^{[12]}$ Stacking more than one type of polar dielectric layers broadens the absorption bands, that is especially useful for radiative cooling. ${ }^{[13]}$ Using single ${ }^{[14]}$ or multiple ${ }^{[1,15]}$ lossless, that is infrared transparent, layers together with polar dielectrics introduce additional design parameters, hence offers better thermal-emission tunability.

Despite the simplicity that the polar dielectrics offer, their emission spectra are limited with the absorption bands. An arbitrary emission spectrum can be achieved by optical resonators such as Salisbury screens that consist of a lossless dielectric layer between a bottom mirror and a thin lossy layer on the top. ${ }^{[16-19]}$ The absorbance wavelengths (resonances) are mainly determined by the optical thickness of the lossless dielectric layer. Alternatively, the lossy dielectric can be coated directly on the mirror layer exhibiting strong interference effects. ${ }^{[20-22]}$ The absorbance wavelengths in this case are primarily determined by the optical properties of the lossy layer and its thickness. The strong interference surfaces consist of a simple bilayer structure, but exhibit broad absorbance spectra. Nanostructured films of metal or lossy materials, e.g., micrometerscale gratings, ${ }^{[23]}$ photonic crystals, ${ }^{[24]}$ antennas, ${ }^{[15,25,26]}$ provide a better control over the emission spectrum at the expense of the ease of fabrication. The emitter design can be as complex 
as patterned photonic crystals atop the lossless/lossy dielectrics stack to achieve the desired optical performance. ${ }^{[27]}$ If the lossy layer exhibits tunable optical properties like graphene, ${ }^{[19,28]}$ quantum dots, ${ }^{[29,30]}$ or phasechange materials, ${ }^{[17,21,22,31,32]}$ the emission spectrum can be dynamically controlled.

Among the aforementioned thermal emitter structures, the ones based on Salisbury screens exploit nanometer-thick absorber/emitter layers, thus present a unique opportunity for optical encryptiondecryption applications by creating patterns that are imperceptible by the naked eye, or cameras, and emit detectable thermal radiation. Such a function defines a new application for thermal emitters that necessitate a new set of design requirements. This study provides a framework for thermal emitters as optical security features and demonstrates applications with various substrates and materials. The ease of fabrication on large-area, flexible substrates like Al foils and imaging by a low-cost smartphone-attachment-type thermal camera using the external temperature of the human body surpasses the performances of the recent reports pursuing alternative approaches such as creating images using plasmonic structures and encryptingdecrypting the images by dynamically controlling the polarization of the incident light ${ }^{[26,33-35]}$ or the optical properties of the structures. ${ }^{[36]}$

\section{Results}

\subsection{Designing the Surfaces}

When an electromagnetic wave is incident on a perfect mirror, standing wave patterns are generated exhibiting periodic electric-field minima and maxima points. Introducing a thin absorber layer at the distance where the field is maximized forms a Salisbury screen and enhances the absorbance by the absorber layer by a factor of $\approx 4$ compared to its absorbance suspended in air. The enhanced infrared absorbance by atop nanometer-thick films with the right choice of materials ${ }^{[37]}$ has led to the idea of revealing the nanometer-thick films that are invisible to the naked eye by sensing their IR emission (Figure 1).

The emission from the surfaces depends on the emissivity and the surface temperature. It is desired to sense the patterns' IR emission close to room temperature $\left(\approx 30^{\circ} \mathrm{C}\right)$ to make use of ubiquitous heat sources like the human body. Thus, the top absorber/emitter material must absorb/emit the IR light around $\lambda=9.5 \mu \mathrm{m}$ that is the peak thermal emission wavelength for a blackbody at $30{ }^{\circ} \mathrm{C}$. The emitter layer must also be optically thin $(n \times t$, where $n$ is the refractive index and $t$ is the thickness) and lossless in the visible such that its presence cannot to be perceived in the visible. Thus, $\mathrm{SiO}_{2}$ is chosen as the absorber/emitter layer due to its low refractive index in the visible, strong absorptivity in the wavelength range of 9-10 $\mu \mathrm{m}$ overlapping with the peak emission wavelength at $30^{\circ} \mathrm{C}$, and ease of deposition (Figure S1, Supporting Information). To enhance the absorbance of the infrared light by the top $\mathrm{SiO}_{2}$ layer, the electric field within this layer, hence at the surface of the dielectric must be enhanced following the Beer's Law. The total electric field on the dielectric surface is the vector sum of electric fields of the incident and reflected rays. For normal incidence, the field-intensity enhancement factor $\left(|E|^{2} /\left|E_{0}\right|^{2}\right)$ is

$\left|1+\frac{r_{12}+r_{23} \mathrm{e}^{2 i \beta}}{1+r_{12} r_{23} \mathrm{e}^{2 i \beta}}\right|^{2}$

where $r_{12}$ is the reflection coefficient for the dielectric-air interface, $r_{23}$ is the reflection coefficient for the metal-dielectric interface, and $\beta$ is the phase-accumulation in the light traveling in the dielectric layer. The total enhancement factor reaches the maximum value of 4 when $\beta$ is $\pi / 2$ and a perfect electrical conductor is used as the mirror that equates $r_{23}$ to -1 . Thus, the reflection coefficient for the mirror layer must be as close as possible to that of a perfect electrical conductor for the maximum enhancement factor. High reflectance from the mirror layer also assures that the absorbance/emissivity of the surface without an emitter layer is low, enhancing the contrast between the IR emissions with and without an emitter layer. In the IR wavelength range of interest (7 to $14 \mu \mathrm{m})$, that is the typical sensitivity range of thermal cameras, $\mathrm{Al}, \mathrm{Au}$, and 


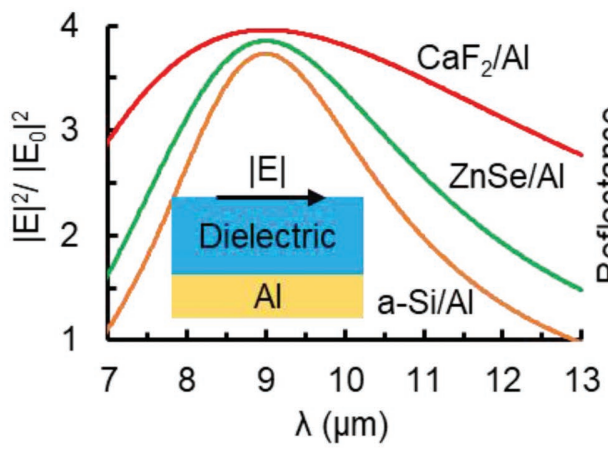

$\lambda(\mu \mathrm{m})$
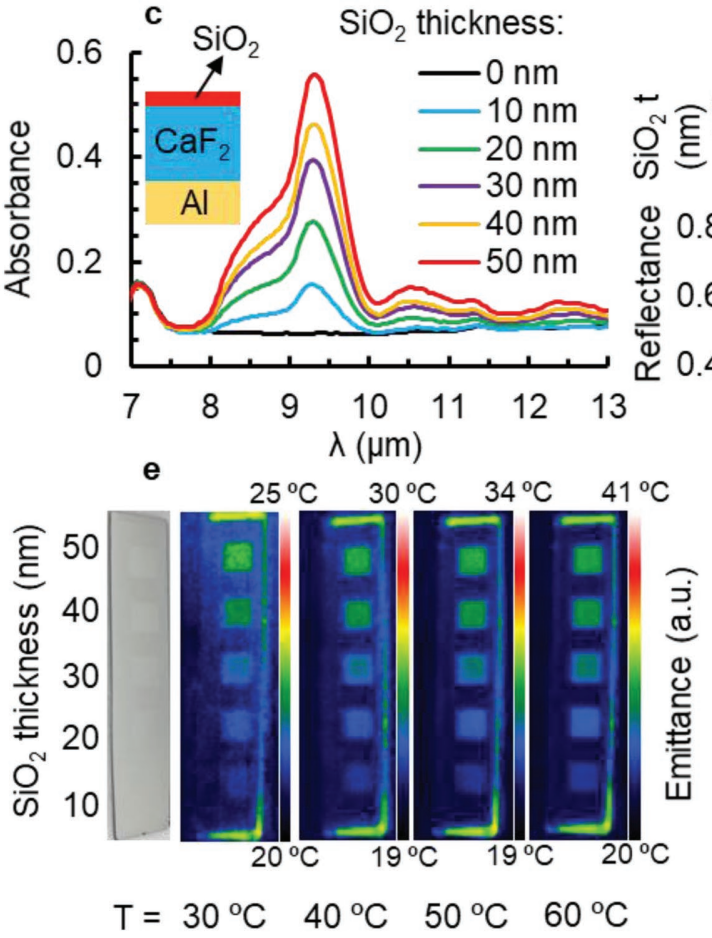

$\mathrm{T}=30^{\circ} \mathrm{C} \quad 40^{\circ} \mathrm{C} \quad 50^{\circ} \mathrm{C} \quad 60^{\circ} \mathrm{C}$ b

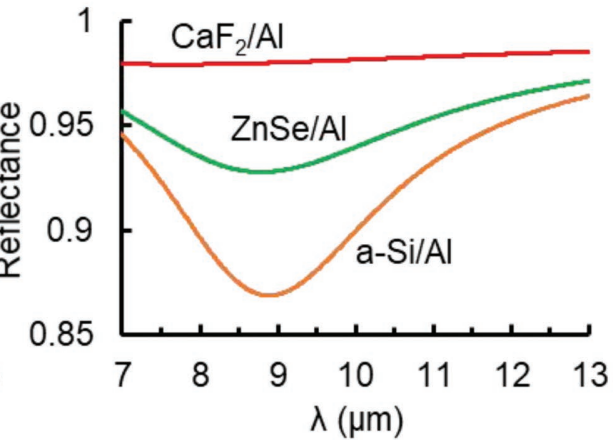

d
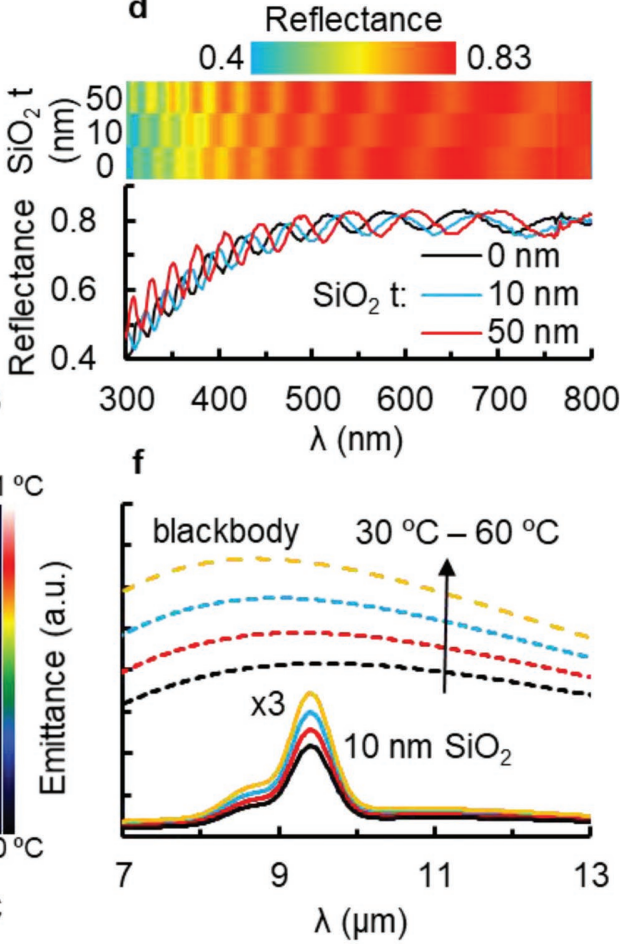

Figure 2. Designing the thermal emitter surfaces. a) Simulated electric field intensity enhancement factors on the dielectric surface for $1772 \mathrm{~nm} \mathrm{CaF}_{2}$, $914 \mathrm{~nm} \mathrm{ZnSe}$, and $661 \mathrm{~nm}$ amorphous Si on Al. The peak enhancement and the enhancement bandwidth are the largest for $\mathrm{CaF}_{2}$, a low refractive index IR-transparent material. The dielectric film thicknesses are adjusted to maximize the field enhancement at $\lambda=9 \mu \mathrm{m}$. b) Simulated reflectance for $\mathrm{CaF}_{2} / \mathrm{Al}, \mathrm{ZnSe} / \mathrm{Al}$, and a-Si/Al surfaces. The peak absorbance/emissivity for bare $\mathrm{CaF}_{2} / \mathrm{Al}$ surface is calculated to be $\approx 2 \%$ in the wavelength range of 7-13 $\mu \mathrm{m}$, whereas it can reach up to 14\% for bare a-Si/Al surface. The refractive indices of the dielectric layers are shown in Figure S5 (Supporting Information). Measured c) absorbance spectra in the infrared and d) reflectance spectra in the visible for $\mathrm{SiO}_{2}$ layers with varying thickness on the field enhancement surface. Color map and plots share the same $x$-axis. e) Photograph and thermal images of $\mathrm{SiO}_{2}$ patches with varying thickness on the field-enhancement surface at elevated temperatures. The patches are formed using a shadow mask and multiple deposition steps. Patch dimensions are $0.5 \times 0.5 \mathrm{~cm}^{2}$. f) Calculated emission spectra for a blackbody (dashed lines) and $10 \mathrm{~nm} \mathrm{SiO}_{2}$ on the field-enhancement surface (solid lines) for the temperature range of $30-60{ }^{\circ} \mathrm{C}$. Emission from $10 \mathrm{~nm} \mathrm{SiO}{ }_{2}$ is scaled by a factor of 3 for clarity.

Ag have the highest reflectance among other metals (Figure S2, Supporting Information). Thus, $\mathrm{Al}$ is chosen as the mirror layer due to its high reflectance in the mid IR and its low cost.

The final stage of the design is choosing the dielectric layer. The most important requirement is being lossless (transparent) in the infrared regime. Commonly used infrared-transparent dielectrics such as $\mathrm{CaF}_{2}$, ZnSe, and $\mathrm{Si}$ are considered for this study. The field intensity enhancement factor is maximized for low refractive-index dielectrics such as $\mathrm{CaF}_{2}$ when the mirror layer is not a perfect electrical conductor but an actual metal (Figure 2a). Furthermore, the bandwidth of the electric field enhancement is the broadest for the material with the lowest refractive index, allowing more tolerance to thickness variations. More importantly, absorption of the incident IR light by the metal layer is lower for low refractive-index dielectrics due to the reduced interaction between the light and the metal layer (Figure 2b, Figures S3 and S4, Supporting Information). The enhanced light-metal interaction results in larger thermal emission from the bare surfaces and worsens the thermal intensity contrast between the background and absorber patterns (Figures S6 and S7, Supporting Information). As a result, $\mathrm{CaF}_{2}$ is chosen as the dielectric material. The approximate 


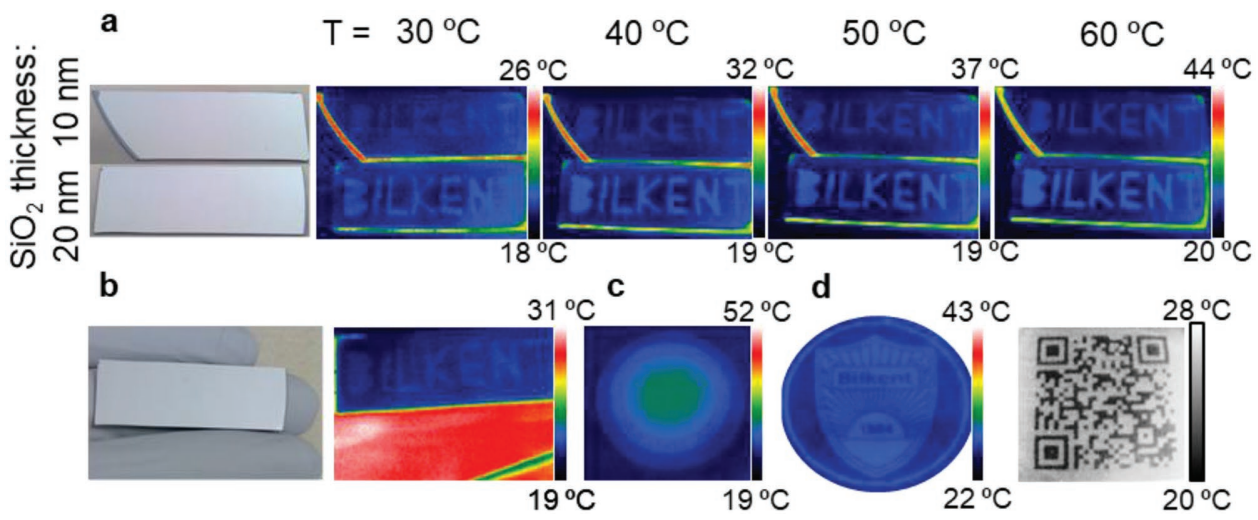

Figure 3. Decrypting patterns in the IR. a) Photographs and thermal images of 10 and $20 \mathrm{~nm}$-thick $\mathrm{SiO}_{2}$ patterns spelling "BILKENT" on the fieldenhancement surfaces. The letter heights are $\approx 1 \mathrm{~cm}$. b) Photograph and thermal image of the sample with $20 \mathrm{~nm} \mathrm{SiO}_{2}$ using the external temperature of the human body. c) Thermal image with 2-bit color depth taken at $60{ }^{\circ} \mathrm{C}$. SiO $\mathrm{Si}_{2}$ thickness for the most outer ring is $5 \mathrm{~nm}$ and increases with $5 \mathrm{~nm}$ steps toward the center. d) Thermal images of $\mathrm{SiO}_{2}$ patterns in the shape of the Bilkent University's logo and a QR code on field-enhancement surfaces. The thermal images are taken at $50{ }^{\circ} \mathrm{C}$. The QR code image is taken in black and white and has enhanced contrast and brightness to be able to scan it using a QR scanner app. The sample sizes are $\approx 3 \times 3 \mathrm{~cm}^{2}$.

dielectric thickness is determined as $\lambda_{\text {peak }} / 4 n_{\mathrm{d}}$, where $\lambda_{\text {peak }}$ is the peak-enhancement wavelength, and $n_{\mathrm{d}}$ is the refractive index of the dielectric layer. The thickness is found as $\approx 1.8 \mu \mathrm{m}$ for $\lambda_{\text {peak }}=9 \mu \mathrm{m}$ using numerical simulations (Figure S8, Supporting Information). As a result, the peak absorbance for atop $10 \mathrm{~nm}$ thick $\mathrm{SiO}_{2}$ is maximized for $\approx 1.8 \mu \mathrm{m}$ thick $\mathrm{CaF}_{2}$. The absorbance by the $\mathrm{SiO}_{2}$ layer decreases for any other $\mathrm{CaF}_{2}$ thickness (Figures S9 and S10, Supporting Information). The peak absorbance calculated for $10 \mathrm{~nm} \mathrm{SiO}_{2}$ on the field-enhancement surface is $\approx 4$ times greater than what is calculated for the same layer suspended in air, following the field-intensity enhancement factor (Figure S1c vs Figure S9, Supporting Information). The final structure is similar to that is designed by Zhen et al. ${ }^{[18]}$ that is $70 \mathrm{~nm} \mathrm{Si}_{3} \mathrm{~N}_{4}$ on 700 a-Si on Al. Zhen et al. has designed their surface to maximize the IR absorbance/emissivity in the wavelength range of $8-13 \mu \mathrm{m}$ (that is the atmospheric transmittance window) for enhanced passivecooling applications, hence the use of a-Si, instead of $\mathrm{CaF}_{2}$, and $70 \mathrm{~nm}$ thick $\mathrm{Si}_{3} \mathrm{~N}_{4}$ as the emitter instead of nanometerthick $\mathrm{SiO}_{2}$. In the rest of the text, $1.8 \mu \mathrm{m}$ thick $\mathrm{CaF}_{2}$-coated $\mathrm{Al}$ mirrors are referred to as the field-enhancement surfaces.

The maximum $\mathrm{SiO}_{2}$ thickness that stays invisible on the field-enhancement surface is determined by experimenting $\mathrm{SiO}_{2}$ thickness in the range of $10-50 \mathrm{~nm}$. The thickest $\mathrm{SiO}_{2}$ film maximizes the thermal emission (Figure S11 in the Supporting Information, Figure 2c). The visible reflectance spectra of the surfaces show interference fringes due to the thick $\mathrm{CaF}_{2}$ that redshift with additional $\mathrm{SiO}_{2}$ layers (Figure 2d). The shift in the reflectance spectra is noticeable for $\mathrm{SiO}_{2}$ films thicker than $20 \mathrm{~nm}$ (Figure 2e). Thus, the $\mathrm{SiO}_{2}$ thickness is chosen to be $20 \mathrm{~nm}$ or lower to hide, or encrypt, the patterns in the visible. The thermal emission from $20 \mathrm{~nm}$ thick $\mathrm{SiO}_{2}$ patch can be distinguished from the background emission at $30{ }^{\circ} \mathrm{C}$. Emissivity of the surfaces multiplied by the blackbody emission spectrum determines the thermal emission from the surfaces (Figure 2f). Hence, the thermal emission from $10 \mathrm{~nm}$-thick $\mathrm{SiO}_{2}$ patch shows a clear contrast with the background for increasing surface temperature, despite being not clear at $30{ }^{\circ} \mathrm{C}$. Elevating the surface temperature is useful when the emitter layer is preferred to be extremely thin, such as $5 \mathrm{~nm}$. Such emitter thicknesses may be required to tolerate the variations in the optical behavior of the field-enhancement surfaces when alternative materials are used, e.g., transparent conductive oxides as the mirror layer.

\subsection{Revealing $\mathrm{SiO}_{2}$ Patterns in the IR}

The lateral dimensions of the $\mathrm{SiO}_{2}$ patterns must be macroscale to be imaged by an ordinary thermal camera without any magnification. The $\mathrm{SiO}_{2}$ patterns are fabricated by stencil method using laser-cut acetate shadow masks. The fieldenhancement surfaces are produced by blanket deposition of $\mathrm{Al}$ and $\mathrm{CaF}_{2}$ layers on 4 " silicon wafers and can potentially be produced on larger areas. Therefore, using the stencil method to form $\mathrm{SiO}_{2}$ patterns makes the whole production process large-area fabrication compatible. The stencil method can also create fine patterns (with $10 \mu \mathrm{m}$ resolution) using electroformed ultrathin metal shadow masks if needed. Such features, however, may not be captured by ordinary thermal cameras. The surfaces are imaged on a hot plate covered with $\mathrm{Al}$ foil to block the IR emission from the plate. Thermal images of $\mathrm{SiO}_{2}$ patterns are observed to be noticeable starting at $\approx 30{ }^{\circ} \mathrm{C}$ for $20 \mathrm{~nm}$ thickness and $\approx 40{ }^{\circ} \mathrm{C}$ for $10 \mathrm{~nm}$ thickness. The emission contrast between the patterns and the background increases with increasing surface temperature for both surfaces (Figure 3a). Revealing the emitter patterns at a lower temperature enables the use of the human body as the heat source (Figure 3b). Using the surrounding that is a few degrees hotter than room temperature as the heat source reduces the thermal imaging setup to only a thermal camera. The thermal camera requirements such as resolution and field of view depend on the resolution and size of the patterns. The images used in this study have critical lateral dimensions on the order of millimeters and have overall sizes of centimeters. Such dimensions are found suitable for the stencil method and as well as thermal imaging of the patterns using a low-cost, smartphone-attachment-type thermal camera with a detector 
a

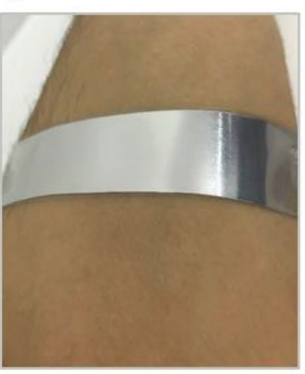

b

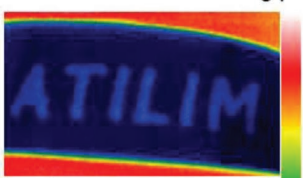

c

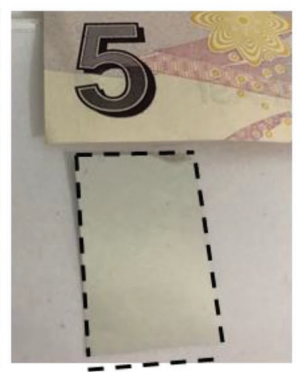

d e

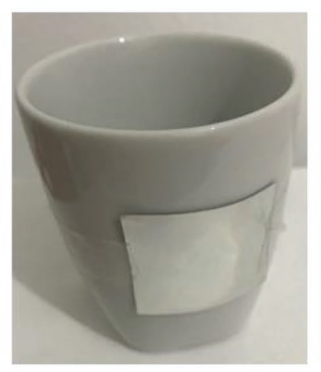

$31^{\circ} \mathrm{C}$

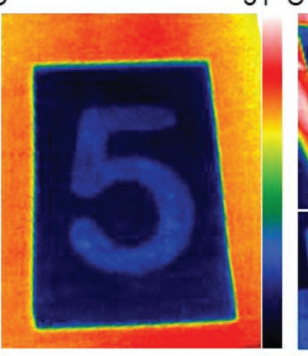

$24^{\circ} \mathrm{C}$

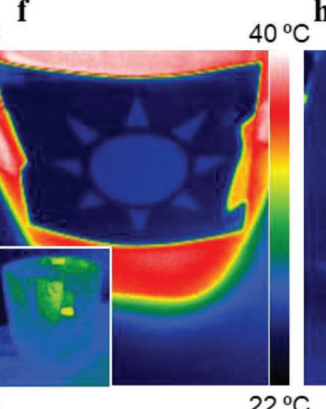

g

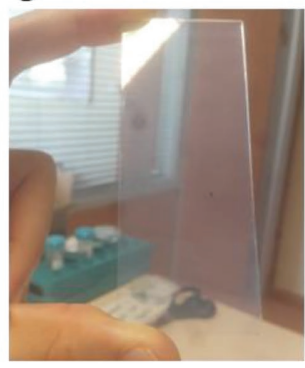

h $32^{\circ} \mathrm{C}$

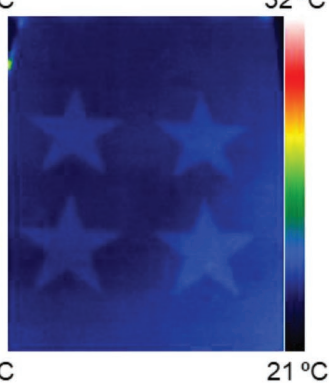

Figure 4. Alternative mirror layers. a) Photograph and b) thermal image of $50 \mathrm{~nm}$ thick $\mathrm{SiO}_{2}$ patterns on $1.8 \mu \mathrm{m} \mathrm{CaF}$-coated $\mathrm{Al}$ foil wrapped around an arm. Thermal images taken before (top) and after (bottom) laminating the foil with a polyethylene film. c) Photograph of a banknote and Al foil substrate (encircled with dashed frame) laminated on a piece of paper. The pattern is generated with $50 \mathrm{~nm}$ thick $\mathrm{SiO}_{2}$. d) The laminated Al foil on paper is wrapped around an arm for thermal imaging. e) Photograph and f) thermal image of $40 \mathrm{~nm}$ thick $\mathrm{SiO}_{2}$ patterns on the field-enhancement surface based on plastic substrate adhered on a mug. Thermal imaging is performed when the mug is empty (inset) and filled with water at $40^{\circ} \mathrm{C}$. The empty mug reflects the thermal emission coming from objects around it. Video S2 (Supporting Information) shows emergence of the thermal image as the mug is filled with hot water. g) Photograph and h) thermal image of $5 \mathrm{~nm}$ thick $\mathrm{SiO}_{2}$ patterns on $1.8 \mu \mathrm{m} \mathrm{CaF}$-coated FTO glass. The thermal image in (h) is taken at $60^{\circ} \mathrm{C}$.

matrix size of $206 \times 156$ pixels. The patterns that are created with single $\mathrm{SiO}_{2}$ deposition step have 1-bit color depth (that is two colors) as shown by Figure 3a,b. Patterns with higher color depth can be created by sequential deposition steps to achieve various $\mathrm{SiO}_{2}$ thicknesses (Figure 3c). If required, the thermal emission from the patterns can also be hidden by covering the surfaces with materials exhibiting strong IR absorbance like polydimethylsiloxane (PDMS) or water. The thermal emission can be concealed permanently by curing PDMS on the surfaces or temporary by simply wetting the surfaces (Figure S12, Supporting Information).

For high resolution $\mathrm{SiO}_{2}$ images, optical lithography is employed. This approach is demonstrated by patterning a photoresist layer on the enhancement surface in the shape of Bilkent University's logo and a QR code followed by depositing $10 \mathrm{~nm} \mathrm{SiO}_{2}$ and lifting off the remaining photoresist (Figure 3d). For a clear contrast between the fine $\mathrm{SiO}_{2}$ features and the background, the surface temperature is brought to $50{ }^{\circ} \mathrm{C}$ for thermal imaging. The QR code is recognized at lower temperatures, but it can only be scanned by the QR code app at elevated temperatures and with enhanced brightness and contrast (Figure S13, Supporting Information).

\subsection{Alternative Mirror Layers}

Switching to bendable substrates from rigid $\mathrm{Si}$ wafers is expected to enhance the versatility of applications. For this task, Al foils are preferred as the bendable substrate as they are inherently low-cost, large area substrates that act like the support and as well as the mirror. The study of varying $\mathrm{SiO}_{2}$ thickness is repeated for $\mathrm{Al}$ foils following deposition of $1.8 \mu \mathrm{m} \mathrm{CaF}$. Visual inspection of the surface reveals that $\mathrm{SiO}_{2}$ patterns as thick as $50 \mathrm{~nm}$ are imperceptible to the naked eye. The thicker $\mathrm{SiO}_{2}$ patterns require only a few degrees above the room temperature to be detected in the thermal images (Figure S14, Video S1, Supporting Information). Al foil's flexibility and tolerance for thicker $\mathrm{SiO}_{2}$ patterns allow wrapping the surfaces on body parts and thermal imaging of the surfaces using the body temperature (Figure 4a). The results demonstrate that thermal imaging of IR emitter patterns is possible on rough substrates after field enhancement (Figure $4 \mathrm{~b})$. In fact, for the application of detecting hidden IR emitter patterns, the rough substrates provide a better camouflage. The mechanical flexibility also enables covering the surface with wear-resistant films. As an example, the Al foil sample is laminated with tens of micrometers thick polyethylene film (Figure $4 \mathrm{~b}$ ). The choice of polyethylene over commonly preferred lamination material polyvinyl chloride (PVC) is based on the polyethylene's high transparency in the wavelength range of interest. Laminating Al foils also enables the use of arbitrary substrates like paper together with $\mathrm{Al}$ foils (Figure 4c,d). One advantage of using arbitrary substrates is the possibility of using the optical security features on banknotes which require many optical security features. Many of the modern banknotes already have metallic features that can also function as the mirror layer for 
a
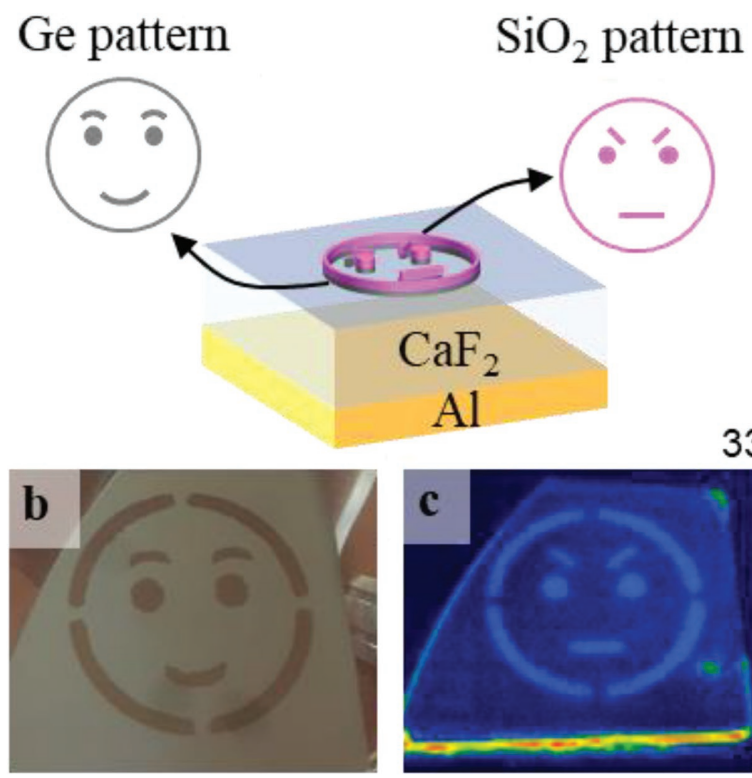

$33^{\circ} \mathrm{C}$

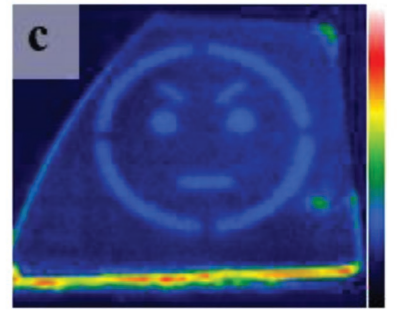

$19^{\circ} \mathrm{C}$

Figure 5. Storing different visual messages in the visible and infrared. a) 3D illustration of the field-enhancement surfaces surface with different patterns generated by $10 \mathrm{~nm} \mathrm{Ge}$ and $10 \mathrm{~nm} \mathrm{SiO}$. b) Photograph of the surface. The text formed by $10 \mathrm{~nm}$ thick $\mathrm{Ge}$ is easily recognizable while the $10 \mathrm{~nm}$ thick $\mathrm{SiO}_{2}$ layer is invisible. c) Thermal image of the surface revealing only the $\mathrm{SiO}_{2}$ pattern at $40{ }^{\circ} \mathrm{C}$.

a security feature using thermal emission. Plastic substrates can also serve as flexible enhancement surfaces after $\mathrm{Al}$ mirror and $\mathrm{CaF}_{2}$ coating (Figure 4e,f). The maximum $\mathrm{SiO}_{2}$ thickness that can be concealed on these substrates, however, is $40 \mathrm{~nm}$. Another important observation is that the infrared absorption performance of the surfaces is unaffected while bending the substrate. This is a direct result of almost angle-independent absorbance/emissivity of the surfaces (Figure S15, Supporting Information).

Another alternative for the mirror layer is transparent conductive oxides that provide optical transparency in the visible while acting like a mirror in the IR (Figure 4g). Fluorine doped tin oxide (FTO) coated glasses are used for the study owing to their high electrical conductivity, hence high optical reflectance in the IR. Varying the $\mathrm{SiO}_{2}$ thickness on $1.8 \mu \mathrm{m}$ $\mathrm{CaF}_{2}$-coated FTO glass reveals that the $\mathrm{SiO}_{2}$ must be $\approx 5 \mathrm{~nm}$ to be imperceptible by the naked eye. The ultrathin $\mathrm{SiO}_{2}$ layer reduces the thermal emission from the $\mathrm{SiO}_{2}$ patterns. Furthermore, FTO exhibits greater absorbance/emissivity compared to the Al mirrors (Figure S16, Supporting Information). Thus, the infrared emission contrast between the $\mathrm{SiO}_{2}$ patterns and the background is weaker for the FTO-based surfaces and can only be observed at higher temperatures $\left(\approx 70{ }^{\circ} \mathrm{C}\right)$ (Figure $\left.4 \mathrm{~h}\right)$. Thin metal (Ag) films are also considered for this application. However, the Ag thicknesses required to achieve acceptable IR performance offer low transparency in the visible, hence overall performance of such surfaces is found inferior to that of the FTO glass substrates (Figure S17, Supporting Information).

\subsection{Storing Different Messages in the Visible and Infrared}

An alternative encryption mechanism is hiding an emitter pattern on top a different visible pattern. This functionality can be achieved using infrared-transparent materials like Ge that are lossy in the visible, hence perceptible by the naked eye. While determining the effect of the refractive index of the dielectric material on the IR performance of the surfaces, it is argued that high refractive-index dielectric materials like Ge increase the background IR emission. On the other hand, if the Ge layer is kept very thin, e.g., $10 \mathrm{~nm}$, on the $\mathrm{CaF}_{2}$ dielectric layer, the generated Ge patterns become visible while the IR emission is not significantly disturbed (Figure S18, Supporting Information). In this mode of operation, the patterns are perceptible by the naked eye and hidden in the infrared that is the opposite of what is demonstrated in the manuscript so far. Encryption is achieved when a different pattern is generated by a thin emitter layer. The patterns detected in the visible and the IR are then different (Figure 5). The performance of the surface is unaffected when the Ge layer is created above the $\mathrm{SiO}_{2}$ pattern (Figure S19, Supporting Information). Alternatively, the silica coating can be present only some part of the visible pattern emphasizing those parts of the visible message in the IR (Figure S20, Supporting Information).

\section{Discussion}

The demonstrated encryption principle is based on materials that are lossless in the visible, but lossy in the infrared such as $\mathrm{SiO}_{2}$. When such a material is coated as nanometer-thick patterns on an infrared-transparent substrate like ZnSe window, it would not be noticed by the naked eye, but would emit IR light. Thus, the pattern would be encrypted in the visible to be decrypted by thermal imaging, albeit requiring high surface temperatures to enhance the IR emission to the detectable levels. In this study, the IR emission from the thin film is enhanced by enhancing its emissivity/absorbance on a uniform field-enhancement surface, enabling decryption of the patterns for relatively low temperatures (Figure S21, Supporting Information). The absorbance enhancement on such a surface follows the electric-field intensity enhancement as described by the Beer's law. ${ }^{[37]}$ Using $\mathrm{Al}$ as the mirror and $\mathrm{CaF}_{2}$ as the dielectric layer provides an enhancement factor of 3.96 that is very close to the theoretical value of 4 . This enhancement factor is much larger than the enhancement factors achieved on simple infrared transparent substrates being $\approx 0.75, \approx 0.35$, and $\approx 0.2$ for $\mathrm{CaF}_{2}$, ZnSe, and Si substrates, respectively, in the wavelength range of interest. As a result, the thermal emission from a $10 \mathrm{~nm}$ thick $\mathrm{SiO}_{2}$ film on the field-enhancement surface at $300 \mathrm{~K}$ is barely matched by the same thickness $\mathrm{SiO}_{2}$ film on a $\mathrm{CaF}_{2}$ substrate at $450 \mathrm{~K}$, which is an impractical temperature for the proposed application (Figure S21, Supporting Information). While the absorber/dielectric/metal structure is kept constant, alternative materials are considered, especially for the metal layer and the substrate, to demonstrate the adaptability of the surfaces (Table S1, Supporting Information).

The field-enhancement can also be achieved using plasmonic structures ${ }^{[38]}$ or photonic crystals, ${ }^{[39]}$ both of which necessitate 
micromachining, challenging the large-area fabrication, hence the ability to use low-cost thermal cameras without magnification. Furthermore, the extreme, but yet, spatially localized field enhancements on such surfaces result in infrared absorbance by the absorber layer comparable to what is observed on the uniform-field enhancement surfaces. ${ }^{[40,41]}$ Another disadvantage of such surfaces would be the increased background thermal emission that would worsen the contrast in thermal images. As a result, the uniform field-enhancement surfaces are preferred over the alternatives like plasmonic structures and photonic crystals.

The optical encryption-decryption function demonstrated in this study exploits the large-area fabrication techniques, low-cost and flexible substrates and a convenient decryption method. These features are superior to those of recently reported alternatives that have either small areas ${ }^{[33-36,42]}$ or involved encryption/decryption methods. ${ }^{[26,43]}$ Table S2 (Supporting Information) lists these alternatives and compares them to this study.

\section{Conclusion}

The results, especially the demonstration of large-area, flexible/bendable substrates, are promising for the potential use of the proposed operation for anticounterfeit applications such as enhancing the optical security features of banknotes.

\section{Experimental Section}

Fabrication: Al was thermally evaporated on $\mathrm{Si}$ or plastic substrates at $\approx 10^{-6}$ Torr at a rate of $\approx 2 \AA \mathrm{s}^{-1} . \mathrm{CaF}_{2}$ was thermally evaporated in the same chamber at a rate of $\approx 20 \AA \mathrm{s}^{-1}$. $\mathrm{CaF}_{2}$ was e-beam evaporated on Al-coated plastic substrate. Ge was thermally evaporated on fieldenhancement surfaces at a rate of $0.5 \AA \mathrm{s}^{-1}$. Ge was also deposited as an adhesion layer on plastic substrate prior to Al deposition. $\mathrm{SiO}_{2}$ was e-beam evaporated on the field enhancement surfaces at a rate of $\approx 2 \AA \mathrm{s}^{-1}$. $\mathrm{SiO}_{2}$ patterns were formed using laser-cut plastic shadow masks. The fine patterns in Figure 4 were achieved using optical lithography and lift-off as described here: The fieldenhancement surfaces on $\mathrm{Si}$ were spin-coated with $\approx 1.4 \mu \mathrm{m}$ thick AZ5214E photoresist $(4000 \mathrm{rpm}, 50 \mathrm{~s})$. The surfaces were prebaked at $110^{\circ} \mathrm{C}$ for $50 \mathrm{~s}$ and exposed with $60 \mathrm{~m}$ ) UV-light using a mask aligner (EVG 620). The photoresist was developed in AZ developer/DI water solution $(1: 4 \mathrm{v} / \mathrm{v})$ for $1 \mathrm{~min}$. The remaining photoresist was lifted-off using acetone following the $\mathrm{SiO}_{2}$ deposition. FTO-coated-glasses were purchased from Solarix.

Preparation of Stencils: $150 \mu \mathrm{m}$ thick clear acetate sheets were laser-cut as stencils. The patterns were created using a CAD software then transferred to a $\mathrm{CO}_{2}$ laser (Epilog, Zing). Laser operated at $10.6 \mu \mathrm{m}$ of wavelength, maximum output power of $30 \mathrm{~W}$ and frequency of $5 \mathrm{kHz}$ with two different cutting modes of raster engraving and vector cutting. The following parameters were used for $\mathrm{CO}_{2}$ laser cutting: Vector cutting mode, $1000 \mathrm{DPI}$ resolution, $4.5 \mathrm{~W}$ output power, $5 \mathrm{kHz}$ frequency, $\approx 100 \mu \mathrm{m}$ beam diameter, and $2.6 \mathrm{~cm} \mathrm{~s}^{-1}$ speed during acetate cutting.

Measurements: Reflection and transmission measurements in the visible and infrared were performed using ellipsometers (J.A. Woollam Co., V-Vase and J.A. Woollam Co., IR-Vase). Thermal images were taken with a Seek Compact thermal camera. Key features of the camera were sensor array size of $206 \times 156$ pixels, $36^{\circ}$ of field of view, spectra range of 7.5-14 $\mu \mathrm{m}$, and focusable lens. The photographs were taken with an iPhone 7.
Simulations: Transfer matrix method was employed to calculate the reflectance, transmittance, and absorbance of surfaces. The refractive index $(n)$ and the extinction coefficient $(k)$ of $\mathrm{CaF}_{2}$ and $\mathrm{SiO}_{2}$ that are used in the simulations are characterized using varying-angle spectroscopic ellipsometer measurements. Palik data were used for the metals.

\section{Supporting Information}

Supporting Information is available from the Wiley Online Library or from the author.

\section{Acknowledgements}

G.B. acknowledges TUBITAK grant \# 114E960.

\section{Conflict of Interest}

The authors declare no conflict of interest.

\section{Keywords}

enhanced infrared absorption, optical security, Salisbury screen, thermal emission, thermal emitters

Received: May 8, 2018

Revised: August 11, 2018

Published online: September 2, 2018

[1] A. Lenert, D. M. Bierman, Y. Nam, W. R. Chan, I. Celanovic, M. Soljacic, E. N. Wang, Nat. Nanotechnol. 2014, 9, 126.

[2] O. Ilic, P. Bermel, G. Chen, J. D. Joannopoulos, I. Celanovic, M. Soljačić, Nat. Nanotechnol. 2016, 11, 320.

[3] K. A. Arpin, M. D. Losego, A. N. Cloud, H. Ning, J. Mallek, N. P. Sergeant, L. Zhu, Z. Yu, B. Kalanyan, G. N. Parsons, G. S. Girolami, J. R. Abelson, S. Fan, P. V. Braun, Nat. Commun. 2013, 4, 2630.

[4] A. P. Raman, M. A. Anoma, L. Zhu, E. Rephaeli, S. Fan, Nature 2014, 515, 540.

[5] P.-C. Hsu, A. Y. Song, P. B. Catrysse, C. Liu, Y. Peng, J. Xie, S. Fan, Y. Cui, Science 2016, 353, 1019.

[6] B. Temelkuran, S. D. Hart, G. Benoit, J. D. Joannopoulos, Y. Fink, Nature 2002, 420, 650

[7] A. Yildirim, M. Vural, M. Yaman, M. Bayindir, Adv. Mater. 2011, 23, 1263.

[8] A. Lochbaum, Y. Fedoryshyn, A. Dorodnyy, U. Koch, C. Hafner, J. Leuthold, ACS Photonics 2017, 4, 1371.

[9] A. Mech, A. Monguzzi, F. Meinardi, J. Mezyk, G. Macchi, R. Tubino, J. Am. Chem. Soc. 2010, 132, 4574.

[10] T. Asano, M. Suemitsu, K. Hashimoto, M. De Zoysa, T. Shibahara, T. Tsutsumi, S. Noda, Sci. Adv. 2016, 2, e1600499.

[11] Y. Zhai, Y. Ma, S. N. David, D. Zhao, R. Lou, G. Tan, R. Yang, X. Yin, Science 2017, 355, 1062.

[12] A. Srinivasan, B. Czapla, J. Mayo, A. Narayanaswamy, Appl. Phys. Lett. 2016, 109, 061905.

[13] J. Kou, Z. Jurado, Z. Chen, S. Fan, A. J. Minnich, ACS Photonics $2017,4,626$.

[14] J. Drevillon, K. Joulain, P. Ben-Abdallah, E. Nefzaoui, J. Appl. Phys. 2011, 109, 034315 
[15] T. Wang, P. Li, D. N. Chigrin, A. J. Giles, F. J. Bezares, O. J. Glembocki, J. D. Caldwell, T. Taubner, ACS Photonics 2017, 4, 1753.

[16] H. Kocer, S. Butun, Z. Li, K. Aydin, Sci. Rep. 2015, 5, 8157.

[17] H. Kocer, S. Butun, E. Palacios, Z. Liu, S. Tongay, D. Fu, K. Wang, J. Wu, K. Aydin, Sci. Rep. 2015, 5, 13384.

[18] Z. Chen, L. Zhu, A. Raman, S. Fan, Nat. Commun. 2016, 7, 13729.

[19] V. W. Brar, M. C. Sherrott, M. S. Jang, S. Kim, L. Kim, M. Choi, L. A. Sweatlock, H. A. Atwater, Nat. Commun. 2015, 6, 7032.

[20] W. Streyer, S. Law, G. Rooney, T. Jacobs, D. Wasserman, Opt. Express 2013, 21, 9113.

[21] M. A. Kats, D. Sharma, J. Lin, P. Genevet, R. Blanchard, Z. Yang, M. M. Qazilbash, D. N. Basov, S. Ramanathan, F. Capasso, Appl. Phys. Lett. 2012, 101, 221101.

[22] M. A. Kats, R. Blanchard, S. Zhang, P. Genevet, C. Ko, S. Ramanathan, F. Capasso, Phys. Rev. X 2013, 3, 041004.

[23] H. Sai, H. Yugami, Appl. Phys. Lett. 2004, 85, 3399.

[24] M. U. Pralle, N. Moelders, M. P. McNeal, I. Puscasu, A. C. Greenwald, J. T. Daly, E. A. Johnson, T. George, D. S. Choi, I. El-Kady, R. Biswas, Appl. Phys. Lett. 2002, 81, 4685.

[25] X. Liu, T. Tyler, T. Starr, A. F. Starr, N. M. Jokerst, W. J. Padilla, Phys. Rev. Lett. 2011, 107, 045901.

[26] M. Makhsiyan, P. Bouchon, J. Jaeck, J.-L. Pelouard, R. Haïdar, Appl. Phys. Lett. 2015, 107, 251103.

[27] E. Rephaeli, A. Raman, S. Fan, Nano Lett. 2013, 130311121615001.

[28] O. Salihoglu, H. B. Uzlu, O. Yakar, S. Aas, O. Balci, N. Kakenov, S. Balci, S. Olcum, S. Süzer, C. Kocabas, Nano Lett. 2018, 18,4541

[29] M. De Zoysa, T. Asano, K. Mochizuki, A. Oskooi, T. Inoue, S. Noda, Nat. Photonics 2012, 6, 535.
[30] T. Inoue, M. De Zoysa, T. Asano, S. Noda, Nat. Mater. 2014, 13, 928.

[31] K.-K. Du, Q. Li, Y.-B. Lyu, J.-C. Ding, Y. Lu, Z.-Y. Cheng, M. Qiu, Light: Sci. Appl. 2016, 6, e16194.

[32] G. Bakan, S. Ayas, E. Ozgur, K. Celebi, A. Dana, ACS Sensors 2016 , 1, 1403.

[33] Y. Cui, R. S. Hegde, I. Y. Phang, H. K. Lee, X. Y. Ling, Nanoscale 2014, 6, 282

[34] F. Yue, C. Zhang, X. Zang, D. Wen, D. B. Gerardot, S. Zhang, X. Chen, Light: Sci. Appl. 2017, 6, e17066.

[35] Q. Dai, M. Ouyang, W. Yuan, J. Li, B. Guo, S. Lan, S. Liu, Q. Zhang, G. Lu, S. Tie, H. Deng, Y. Xu, M. Gu, Adv. Mater. 2017, 1701918.

[36] X. Duan, S. Kamin, N. Liu, Nat. Commun. 2017, 8, 14606.

[37] S. Ayas, G. Bakan, E. Ozgur, K. Celebi, A. Dana, ACS Photonics 2016 , $3,337$.

[38] C. Ciracì, R. T. Hill, J. J. Mock, Y. Urzhumov, A. I. FernándezDomínguez, S. A. Maier, J. B. Pendry, A. Chilkoti, D. R. Smith, Science 2012, 337, 1072.

[39] N. Ganesh, W. Zhang, P. C. Mathias, E. Chow, J. A. N. T. Soares, V. Malyarchuk, A. D. Smith, B. T. Cunningham, Nat. Nanotechnol. 2007, 2, 515.

[40] C. Huck, A. Toma, F. Neubrech, M. Chirumamilla, J. Vogt, F. De Angelis, A. Pucci, ACS Photonics 2015, 2, 497.

[41] J. M. Hoffmann, H. Janssen, D. N. Chigrin, T. Taubner, Opt. Express 2014, 22, 14425

[42] J. Xue, Z.-K. Zhou, Z. Wei, R. Su, J. Lai, J. Li, C. Li, T. Zhang, X.-H. Wang, Nat. Commun. 2015, 6, 8906.

[43] C. Zhang, B. Wang, W. Li, S. Huang, L. Kong, Z. Li, L. Li, Nat. Commun. 2017, 8, 1138 . 